\title{
Modeling TCP and High Speed TCP: A Nonlinear Extension to AIMD Mechanisms
}

\author{
Richard Marquez ${ }^{1,3}$, Eitan Altman ${ }^{2}$, and Solazver Solé-Álvarez ${ }^{1}$ \\ 1 Postgrado en Ingeniería de Control, Facultad de Ingeniería \\ Universidad de Los Andes, Mérida 5101, Venezuela \\ \{marquez, solazver\}@ula.ve \\ 2 INRIA BP93, 06902 Sophia Antipolis, France \\ altman@sophia.inria.fr \\ 3 Departamento de Sistemas de Control, Facultad de Ingeniería \\ Universidad de Los Andes, Mérida 5101, Venezuela
}

\begin{abstract}
We propose two modeling approaches of AIMD congestion control mechanisms. The first separates the increase and decrease parts where as the second describes the rate evolution as a continuous process governed by a differential equation. We relate the approaches and show that the second one is an averaged approximation of the first one. The objective of this paper is twofold: model a class of (stable) generalized TCP protocols as a nonlinear extension of AIMD mechanisms, and in particular to analyze stability of Floyd's HighSpeed TCP. The class of models studied will be useful for control design and tuning.
\end{abstract}

\section{Introduction}

TCP regulates its congestion window by an additive increase/multiplicative decrease (AIMD) mechanism usually represented by parameters $a$ and $b$, respectively, see $[1,2]$. Many authors indicate that TCP performance is poor when fast long-distance networks are considered, cf. [3,4]. Floyd et al. [4] proposed HighSpeed TCP, a modification of TCP basic algorithm, designed to fit some requirements of high speed networks. They propose increase/decrease parameters $a(w)$ and $b(w)$ which depend on window size $w$.

We study two modeling approaches for these AIMD mechanisms. The "discontinuous model" separates the increase and decrease parts; it uses smooth increase of the rate with sharp smoothed decrease at loss instants, see e.g. [5, 6 . The second approach describes the window evolution as a continuous process governed by a differential equation, a so-called fluid model, see e.g. $[7,8,6]$. Our first goal is to make the relation between these two approaches. We propose a class of generalized TCP (GTCP) of which standard TCP and HSTCP are special cases, and obtain qualitative stability properties: the existence of a unique periodic orbit process to which the window size converges from any initial value. Whereas similar type of results have been known for TCP, this type of result is new in the context of HSTCP, and not at all obvious, as we know of cases of 
several limiting regimes that may occur when $a$ and $b$ depend on $w$ (in particular when $a$ increases in $w$ ), cf. [9, Remark 2].

The structure of the paper is as follows. In Section 2, we review briefly TCP algorithm, the discontinuous TCP model is then deduced. Section 3 presents our modeling assumptions and applies basic results of averaging theory to the proposed discontinuous TCP model. Comparisons of TCP models, and NS simulations are provided, confirming theoretical findings. A class of (stable) generalized TCP models are proposed in Section 4 as a nonlinear extension of AIMD congestion control mechanisms. Uniqueness conditions and asymptotic stability results are derived for GTCP. We finish with some conclusions and remarks.

\section{Discontinuous Nature of TCP}

In this section, a "binary feedback" model of TCP, represented by a discontinuous differential equation, is derived.

\section{TCP Congestion Control Mechanism.}

Consider a single source. Let $u$ denote the congestion indication signal. This variable represents the binary feedback of [1], where $u=0$ represents the noncongestion (increase load) phase, and $u=1$ indicates the decrease part due to packet losses.

TCP congestion window behavior can be explained in the following form. Assume a discrete time mechanism with time divided into round-trip times (RTTs). In congestion avoidance, if there is no congestion, that is $u=0$, the congestion window cwnd is increased by $a$ every time a full window is acknowledged (after a RTT), this phase is known as additive increase,

$$
\text { ACK: } \quad \operatorname{cwnd}[n+1]=\operatorname{cwnd}[n]+a
$$

After a congestion is detected ${ }^{1}$, denoted here by $u=1$, the cwnd is reduced by a factor $1-b$ (multiplicative decrease):

$$
\text { Drop: } \quad \operatorname{cwnd}[n+1]=(1-b) \operatorname{cwnd}[n]
$$

In particular, we consider a NewReno-like implementation of TCP which reduces its congestion window at most once per RTT. Usually, $a=1, b=1 / 2$ are the canonical values for TCP. In general, we refer to pure AIMD congestion control by $\operatorname{AIMD}(a, b)$, with increase parameter $a$, and decrease parameter $b$. Let us analyze this behavior.

\section{Discontinuous TCP Model.}

Increasing Part: Dividing (1) by RTT, this equation can be rewritten as the rate of change of the congestion window size, as follows

$$
\frac{\operatorname{cwnd}[n+1]-\operatorname{cwnd}[n]}{\mathrm{RTT}}=\frac{a}{\mathrm{RTT}}
$$

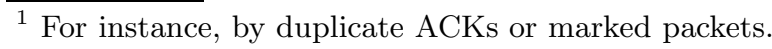


Thus, the left-hand side approximates the derivative of the window size. Replacing cwnd by the continuous variable $w$, it yields the standard TCP differential equation of the additive increase phase $[10,7]$ :

$$
\frac{d w}{d t}=\frac{a}{\mathrm{RTT}}
$$

Decreasing Part: Instead of considering jumps at loss instants, we prefer to smooth the jumps over the RTT duration, which reflects the fact that in practice the throughput does not decrease immediately. This is also in line with modeling of the decrease part in other congestion control protocols $[11]^{2}$. Thus, we approximate (2) by the continuous-time differential equation

$$
\frac{d w}{d t}=-\frac{k}{\mathrm{RTT}} w
$$

which holds for a duration of RTT.

Let us analyze the last equation with respect to (2). Beginning with a window size $w_{0}$, the congestion window decreases, after one RTT to $w(t=\mathrm{RTT})=(1-$ b) $w_{0}$. A solution to (4) is given by

$$
w(t)=w_{0} \exp (-k t / \text { RTT })
$$

where $w_{0}=w(0)$. Therefore, we have $(1-b) w_{0}=w_{0} \exp (-k)$ which results in $k=-\log (1-b)$.

Note, $-\log (1-b)=\frac{2 b}{2-b}+O\left(\left(\frac{2 b}{2-b}\right)^{3}\right)$. In later calculations, particularly in the analysis of Floyd's HSTCP, it will be more appropriate to approximate $-\log (1-b)$ by $2 b /(2-b)$ on the interval $0 \leq b \leq 0.5$. Equation (4) then becomes ${ }^{3}$

$$
\frac{d w}{d t}=-\frac{2 b}{2-b} \frac{w}{\mathrm{RTT}}
$$

Resulting 'Binary Feedback' Model. Congestion signal $u$, which takes values on $\{0,1\}$, can be used to bring together Equations (3) and (5), leading to a discontinuous differential equation ${ }^{4}$ model:

$$
\frac{d w}{d t}=\frac{a}{\mathrm{RTT}}(1-u)-\frac{2 b}{2-b} \frac{w}{\mathrm{RTT}} u
$$

or, equivalently,

$$
\operatorname{RTT} \frac{d w}{d t}=a-\left(a+\frac{2 b}{2-b} w\right) u
$$

with $w_{0}=w(0) \geq 1$. It is easy to see that when $u=0$ we obtain (3), and (5) otherwise.

${ }^{2}$ Nonetheless, as we will later our approach relies on the basic reasonable assumption RTT $\ll T$ (where $T$ is the average time between losses), providing a reliable approximation to instantaneous jumps.

3 The term $2 b /(2-b)$, that is, a factor of $2 / 3$ when $b=1 / 2$, appears explicitly in Reno-1, and Reno-2 models proposed in [12].

4 This kind of models reminds the variable structure systems of Utkin [13]. Reference [14] presents technical details and methods for analysis of this class of differential equations. 


\section{Averaged TCP Model}

\subsection{Assumptions}

In the sequel, we consider the following assumptions:

1. A constant inter-loss time $T$, and

2. A fixed round trip time RTT much smaller that $T$, i.e., RTT $\ll T$, such that quotient RTT/ $T$ is constant.

Note that in practice the inter-loss time $T$ is often random (e.g. in when TCP operates over wireless channels). We prefer to approximate it by a constant (that may be related to an expected inter-loss time). This is a standard approximation in the modeling of TCP, see e.g. [15] as well as [2] where, using a deterministic inter-loss model, AIMD response function is derived. If random independent inter-loss times are replaced by their expectation, then the steady state throughput decreases, see [16, eq 9]. Thus the model we study can be used as a bound.

Assumption 1 leads to the following scalar control function $u=u(t)$ :

$$
u(t)=\left\{\begin{array}{lll}
0 & \text { if } & t_{k} \leq t<t_{k}+(T-\mathrm{RTT}) \\
1 & \text { if } \quad t_{k}+(T-\mathrm{RTT}) \leq t<t_{k+1}
\end{array}\right.
$$

where $t_{k}$ is the time instant when additive increase begins. A congestion epoch, given by $\left[t_{k}, t_{k+1}\right]$, has a duration $T=t_{k+1}-t_{k}$. Function $u(t)$ is then $T$-periodic, i.e., $u(t+T)=u(t)$ for all $t$.

Taking into account the periodic behavior of (6) under the control signal (7), we will apply the method of averaging. In the study of systems which combine slow and fast motions, the averaging principle suggests that a good approximation of the slow motion on long time intervals can be obtained by averaging its parameters in fast variables. This approach is standard in the case of, for example, control of DC-to-DC converters [17]: the discontinuous model is translated into a model of continuous nature, easier to analyze from a control theory point of view.

\section{$3.2 \quad$ Averaging}

We first summarize some basic results on averaging theory, which are taken from Sanders and Verhulst [18] and from Khalil [19].

Let $x, y, x_{0}$ belong to an open subset $D \subset \mathbf{R}$, let $t \in \mathbf{R}^{+}=[0, \infty)$, and let the parameter $\epsilon$ vary in the range $\left(0, \epsilon_{0}\right]$ with $\epsilon_{0} \ll 1$. Let $f: \mathbf{R}^{+} \times D \rightarrow \mathbf{R}$ be a piecewise continuous function ${ }^{5}$. Consider the initial value problem

$$
\frac{d x}{d t}=\epsilon f(t, x), \quad x(0)=x_{0}
$$

\footnotetext{
${ }^{5}$ Here the classical "smooth" assumption is replaced by a "piecewise continuous" assumption, this approach is justified by [20, Appendix C] to define the concept of solution of a differential equation. Another approach consists in regarding (6)-(7) as a Carathéodory differential equation [14, Chap. 1].
} 
If $f(t, x)$ is a $T$-periodic function in its first argument, we let the averaged system be

$$
\begin{gathered}
\frac{d y}{d t}=\epsilon f^{0}(y), \quad y(0)=x_{0} \\
f^{0}(y)=\frac{1}{T} \int_{0}^{T} f(t, y) d t
\end{gathered}
$$

From $[18$, p.39,71] and [19, p.430], we have the following

Theorem 1. There exists a positive $\epsilon_{0}$ such that, for all $0<\epsilon \leq \epsilon_{0}$,

1. $x(t)-y(t)=O(\epsilon)$ as $\epsilon \rightarrow 0$ on the time scale $1 / \epsilon$, and

2. if $x^{*}$ is a hyperbolically (resp. asymptotically) stable equilibrium ${ }^{6}$ point for $f^{0}$, then $x(t)-y(t)=O(\epsilon)$ as $\epsilon \rightarrow 0$ for all $t \in \mathbf{R}^{+}$, and the differential equation (8) possesses a unique periodic orbit (periodic solution) which is hyperbolically (resp. asymptotically) stable and belongs to an $O(\epsilon)$ neighborhood of $x^{*}$.

Consider the following averaged quantities:

Definition 1. Time-average congestion window $\bar{w}$ and averaged control $\mu$ are defined, respectively, by

$$
\bar{w}=\frac{1}{T} \int_{0}^{T} w(\sigma) d \sigma \quad \text { and } \quad \mu=\frac{1}{T} \int_{0}^{T} u(\sigma) d \sigma
$$

We use $\bar{w}$ to denote the average de $w$ over an entire congestion epoch. Expression (11) and previous assumptions lead to a constant $\mu$, independent of time scaling, given by

$$
\mu=\frac{\mathrm{RTT}}{T}
$$

Note $\mu$ corresponds to a sort of normalized packet-loss rate.

Equations (6) and (7) can be represented as a standard averaging problem by changing the time variable from $t$ to $s=\epsilon t$, that is:

$$
\begin{aligned}
\operatorname{RTT} \frac{d w}{d s} & =\epsilon\left[a-\left(a+\frac{2 b}{2-b} w(s)\right) u(s)\right] \\
u(s) & =\left\{\begin{array}{lll}
0 & \text { if } & s_{k} \leq s<s_{k}+\frac{(T-\mathrm{RTT})}{\epsilon} \\
1 & \text { if } & s_{k}+\frac{(T-\mathrm{RTT})}{\epsilon} \leq s<s_{k+1}
\end{array}\right.
\end{aligned}
$$

where $s_{k}=t_{k} / \epsilon$. Control input $u(s)$ is periodic in $s$ of period $T / \epsilon$. A reasonable choice of $\epsilon$ is $\epsilon=\alpha$ RTT, $0<\alpha<1$.

Thus, we associate with (11) the autonomous averaged system

$$
\operatorname{RTT} \frac{d \bar{w}}{d s}=\epsilon\left[a-\left(a+\frac{2 b}{2-b} \bar{w}\right) \mu\right]
$$

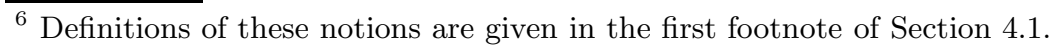


where $u(s)$ has been replaced by its average $\mu$. Note that $\mu$ must be necessarily limited to $0 \leq \mu=\mathrm{RTT} / T \leq 1$.

Theorem 1 justifies approximating the solutions $w(t)$ of of the non-autonomous system (11) by the solutions $\bar{w}(t)$ of the averaged system (12). Moreover, for $\mu=0$ or 1 , there is no distinction between (11) and (12). Averaged system (12) has a unique equilibrium point $\bar{w}^{*}=\frac{a(2-b)}{2 b}\left(\frac{1}{\mu}-1\right)>1$, which is asymptotically stable (a.s.). To show this, define a Lyapunov function $V(\bar{w})=1 / 2\left(\bar{w}-\frac{a(2-b)}{2 b}\left(\frac{1}{\mu}-1\right)\right)^{2}$, yielding $\dot{V}(\bar{w})<0$ for all $\bar{w}>1$ except for $\bar{w}=\bar{w}^{*}$. Thus, system (11) possesses an a.s. periodic solution of period $T / \epsilon$.

Pulling back to original time coordinates, our TCP averaged model is given by

$$
\operatorname{RTT} \frac{d \bar{w}}{d t}=\left[a-\left(a+\frac{2 b}{2-b} \bar{w}\right) \mu\right]
$$

In Figure 1, a numerical simulation is depicted comparing the discrete behavior (1)-(2), and the response of discontinuous (6)-(7), and averaged (13) TCP models. Notice that the discontinuous and averaged system trajectories fit well the (periodic) discrete dynamical behavior of TCP. In steady state oscillation, the discontinuous model response agrees satisfactorily with the deterministic analysis of [2]. The initial condition of the averaged differential equation is taken to be $\bar{w}(0)=w(0)$. The average window is suitably predicted by the proposed model, compared to an NS simulation obtained under similar conditions.
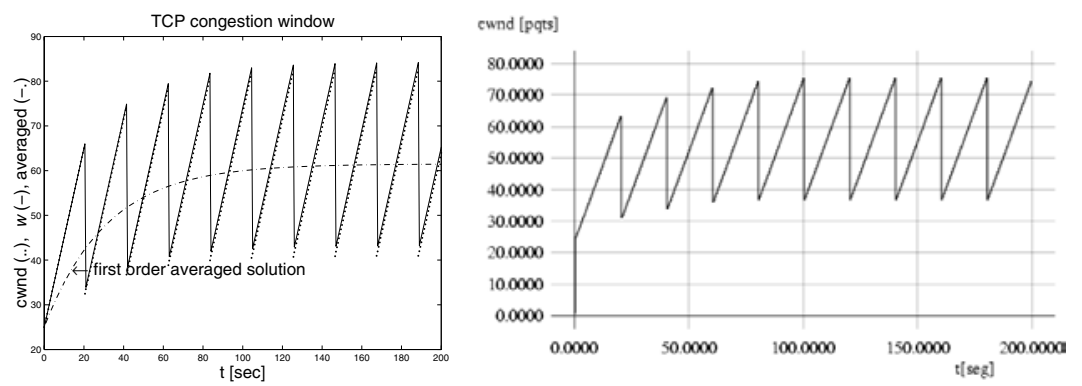

Fig. 1. left: the discrete behavior (dotted line) which coincides with the discontinuous model (solid line), and the averaged (dash-dotted) TCP window size, initial condition cwnd $=w(0)=\bar{w}(0)=25$ packets, $T=21 \mathrm{sec}$, RTT $=0.5 \mathrm{sec}$. NS simulation (right) with constant inter-loss time.

\subsection{Further Analysis}

Second-Order Averaging. In order to show that the averaging principle fits well to our purposes, let us consider the second-order averaging. Define $\phi(t)=$ $\operatorname{RTT} \mu / 2-\int_{0}^{t}[u(\sigma)-\mu] d \sigma$; it is $T$-periodic and bounded for $t \geq 0$. A second-order averaged solution $\bar{w}^{S}$ to $(6)-(7)$ is given by 


$$
\bar{w}^{S}(t)=\bar{w}(t)-\frac{1}{\mathrm{RTT}}\left(a+\frac{2 b}{2-b} \bar{w}(t)\right) \phi(t)
$$

where $\bar{w}(t)$ is a solution of (13). Furthermore, taking $t=0, \bar{w}^{S}(0)=w(0)$ and solving for $\bar{w}(0)$, a corrected estimate of $\bar{w}(0)$ results from (14),

$$
\bar{w}(0)=\frac{a\left(\frac{1}{2} \mu\right)+\bar{w}^{S}(0)}{1+\frac{2 b}{2-b}\left(-\frac{1}{2} \mu\right)}=\frac{a\left(\frac{1}{2} \mu\right)+w(0)}{1-\frac{2 b}{2-b}\left(\frac{1}{2} \mu\right)}
$$

Figure 2 compares discontinuous (6), first- (13), and second-order (14) averaged TCP solutions, considering the corrected initial condition (15). The corrected initial condition produces a solution which follows closely moving averages along the original periodic TCP trajectories.
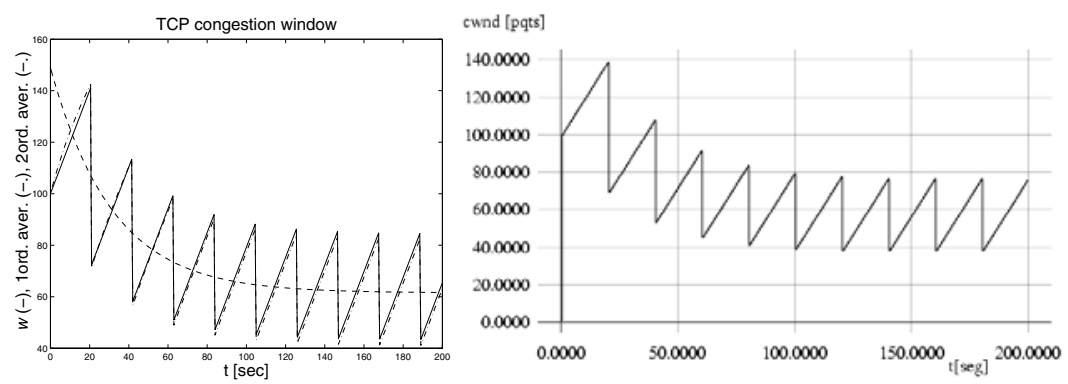

Fig. 2. Comparison (left) of discontinuous (solid), first- (dashed), and second-order (dash-dotted) averaged TCP solutions, $w(0)=100$ packets, $\bar{w}(0)=148.96$ packets (corrected initial condition), $T=21 \mathrm{sec}, \mathrm{RTT}=0.5 \mathrm{sec}$. NS simulation (right).

Steady State TCP Behavior. See [2]. In terms of $T$, the mean $W$ of the TCP congestion window in steady state, results in

$$
W=\frac{a(2-b)}{2 b} \frac{T}{\mathrm{RTT}}
$$

The total number of packets between losses is $n=W \times T /$ RTT; the per-packet drop rate $p$ is $n=1 / p$, i.e. $p T W=$ RTT. Hence,

$$
p=\frac{a(2-b)}{2 b} \frac{1}{W^{2}}
$$

Model (13) depends on the normalized packet-loss rate $\mu$. It can also be written in terms of $p$. Replacing $W$ by $\bar{w},(10)$ yields $\mu=p \bar{w}$. Hence, (13) becomes

$$
\operatorname{RTT} \frac{d \bar{w}}{d t}=a-\left(a+\frac{2 b}{2-b} \bar{w}\right) \bar{w} p
$$


where $p$ is a new 'control' parameter. Let us analyze this system for a fixed averaged window size $\bar{w}^{*}$. In steady-state, the equilibrium point $\left(\bar{w}^{*}, p^{*}\left(\bar{w}^{*}\right)\right)$ yields

$$
p^{*}=\frac{a}{\left(a+\frac{2 b}{2-b} \bar{w}^{*}\right) \bar{w}^{*}}
$$

When $\bar{w}^{*}$ is large, $\frac{2 b \bar{w}^{*}}{2-b} \gg a$, equation (19) translates into (17).

Remark 1. Consider similarities of (18) to fluid-flow models presented in the literature, cf. for instance [7,8]; observe that time delays and time-dependent RTTs would be easily incorporated in our analysis. Note, moreover, the model in [8] is also obtained by "averaging": it is an averaging over many samples, i.e. the statistical expectation that is used. In this paper, in contrast, we use timeaveraging over a single sample path. Similar (time-)averaging could be used also for each individual sample in models with random time between losses.

\section{Generalized TCP Protocols}

AIMD algorithms are included in a class of so-called linear controls defined in [1]. In this section, the modeling approach presented above serves to establish a large class of nonlinear AIMD mechanisms. Thus, generalized TCP (GTCP) models are proposed under appropriate conditions, namely (local) asymptotic stability of an (sometimes unique) equilibrium point of averaged GTCP models. We show that standard TCP and HSTCP models presented in this paper are particular cases of these generalized models.

\subsection{Main Result}

Let us define a discontinuous GTCP model as

$$
\operatorname{RTT} \frac{d w}{d t}=\mathrm{g}(w)-(\mathrm{g}(w)+w \mathrm{~h}(w)) u
$$

where $w$ is the congestion window size, and $u$ is given as before by $(7) ; \mathrm{g}(w)$, $\mathrm{h}(w)$ are appropriate smooth functions defining, resp., increase and decrease behaviors. In fact, these functions define the increase/decrease parameters $a(w)$, $b(w)$ which depend on the present window size $w$.

Averaged GTCP model is thus given by

$$
\operatorname{RTT} \frac{d \bar{w}}{d t}=\phi(\bar{w}, \mu)=\mathrm{g}(\bar{w})-(\mathrm{g}(\bar{w})+\bar{w} \mathrm{~h}(\bar{w})) \mu
$$

where congestion signal $u$ have been replaced by the normalized packet-loss rate $\mu$. At equilibrium $\phi\left(\bar{w}^{*}, \mu\right)=0$, that is,

$$
\mu=\frac{\mathrm{g}\left(\bar{w}^{*}\right)}{\mathrm{g}\left(\bar{w}^{*}\right)+\bar{w}^{*} \mathrm{~h}\left(\bar{w}^{*}\right)}=\lambda\left(\bar{w}^{*}\right)
$$


where $\lambda:(1, \infty) \rightarrow(0,1)$. A (locally) (a.s.) equilibrium point ${ }^{7}$ exists if the Jacobian of $f$ satisfies

$$
\frac{\partial \phi}{\partial \bar{w}}\left(\bar{w}^{*}, \mu\right)<0
$$

In our setting, Theorem 1 yields the following

Lemma 1. The discontinuous GTCP model (20) possesses a unique hyperbolic periodic orbit if the following conditions are satisfied $\forall w>1$ :

1. $\mathrm{g}(w)>0$,

2. $\mathrm{h}(w)>0$,

3. the invertible function $\lambda^{-1}:(0,1) \rightarrow(1, \infty)$ exists, and

4. $-\mathrm{h}(w) \mathrm{g}(w)-w\left[\mathrm{~g}(x) \frac{\partial \mathrm{h}}{\partial w}-\mathrm{h}(x) \frac{\partial \mathrm{g}}{\partial w}\right]<0$.

Proof. Straightforward by applying Theorem 1. Replace $x^{*}$ by $w^{*}, x$ by $w, y$ by $\bar{w}, x_{0}$ by $w_{0}$. Condition 3 and 4 guarantees, resp., the existence of a (unique) equilibrium point and asymptotic stability $(22)^{8}$.

The following result includes the class of linear AIMD algorithms, cf. [1,2]:

Corollary 1. Discontinuous TCP (6)-(7) possesses a unique hyperbolic periodic orbit.

Proof. Define $\mathrm{g}(w)=a, \mathrm{~h}(x)=2 b /(2-b)$; a.s. equilibrium point of (13) is given by

$$
\bar{w}^{*}=\frac{a(2-b)}{2 b} \frac{1-\mu}{\mu}=\frac{a(2-b)}{2 b}\left(\frac{T}{\mathrm{RTT}}-1\right)
$$

\subsection{HighSpeed TCP}

Conditions 1-4 of Lemma 1 serves to analyze and design appropriate nonlinear AIMD congestion control mechanisms, in particular in order to define suitable $a(w)$ and $b(w)$ parameters. This is the case of HSTCP. HSTCP is described as

${ }^{7}$ Let us recall the following Theorem [19, Th. 4.7]: Let $x=x^{*}$ be an equilibrium point for the nonlinear system $\dot{x}=f(x)$ where $f: D \longrightarrow R^{n}$ is continuously differentiable and $D$ is a neighborhood of $x^{*}$. Let

$$
A=\left.\frac{\partial f}{\partial x}(x)\right|_{x=x^{*}}
$$

Then,

1. $x^{*}$ is asymptotically stable (denoted a.s.) if the real part $\Re\left(z_{i}\right)<0$ for all eigenvalues $z_{i}$ of $A$.

2. $x^{*}$ is unstable if $\Re\left(z_{i}\right)>0$ for one or more $z_{i}$ of $A$.

In this context, an equilibrium point is called hyperbolic if $\Re\left(z_{i}\right) \neq 0$. Asymptotic stability means $\lim _{t \rightarrow \infty} x(t)=x^{*}$. Roughly speaking, a hyperbolic periodic orbit is analogous to that of a hyperbolic equilibrium point.

8 Observe proposed conditions are similar to those implied by sliding regimes of nonlinear variable-structure feedback systems, cf. [13]. 
follows [4]. When the window size is smaller than a given value $\omega$, i.e. cwnd $\leq \omega$, the increase/decrease parameter functions are $a$ (cwnd) $=a=1, b$ (cwnd) $=b=$ $1 / 2$, as in standard TCP. For cwnd $>\omega$, parameter $\pi$ (cwnd) is defined, which is reminiscent of the per-packet drop rate $p$. Thus, HSTCP parameters are given by

$$
\begin{aligned}
& \pi(\text { cwnd })=e^{\left[\log \left(P_{1}\right)-\log (P)\right] \frac{\log (\text { cwnd })-\log (\omega)}{\log \left(\omega_{1}\right)-\log (\omega)}+\log (P)} \\
& b(\text { cwnd })=(B-b) \frac{\log (\text { cwnd })-\log (\omega)}{\log \left(\omega_{1}\right)-\log (\omega)}+b \\
& a(\text { cwnd })=\text { cwnd }^{2} \pi(\text { cwnd }) \frac{2 b(\text { cwnd })}{2-b(\text { cwnd })}
\end{aligned}
$$

where 'log' is the natural logarithm. Default values are: $B=0.1, \omega=31$, $P=3 /\left(2 \omega^{2}\right), P_{1}=10^{-7}, \omega_{1}=83000$.

Discontinuous HighSpeed TCP model is given by $(20)$, where $\mathrm{g}(w)$ and $\mathrm{h}(w)$ are given by

$$
\mathrm{h}(w)=\frac{2 b(w)}{2-b(w)}, \quad \mathrm{g}(w)=a(w)=w \pi(w) \mathrm{h}(w)
$$

where cwnd has been replaced by $w, a(w), b(w)$ are defined by $(24)$.

The following result is not at all obvious for this nonlinear extension of AIMD algorithms:

Corollary 2. Discontinuous HSTCP model defined by (25) possesses a unique hyperbolic periodic orbit for all initial conditions $w_{0}>1$ that satisfies $b\left(w_{0}\right)>0$.

Proof. Conditions 1-4 of Lemma 1 apply for initial condition $\omega<w_{0}<\bar{\omega}=$ 597045, where $b\left(w_{0}\right)>0$. Parameters (24) implies $\mathrm{g}(\bar{w}), \mathrm{h}(\bar{w})>0$, for $\omega<\bar{w}<$ $\bar{\omega}$. Thus, $\bar{w}^{*} \pi\left(\bar{w}^{*}\right)-\left(\bar{w}^{*} \pi\left(\bar{w}^{*}\right)+1\right) \mu=0$. Function $\lambda^{-1}$, for $\omega<\bar{w}^{*}<\bar{\omega}$,

$$
\bar{w}^{*}=k_{1}\left(\frac{1-\mu}{\mu}\right)^{k_{2}}=\lambda^{-1}(\mu)
$$

where $k_{1}=e^{\left(\frac{\log \left(P_{1}\right) \log (\omega)-\log (P) \log \left(\omega_{1}\right)}{\log \left(\omega_{1}\right)-\log (\omega)+\log \left(P_{1}\right)-\log (P)}\right)}$, and $k_{2}=\log _{\frac{\omega_{1} P_{1}}{\omega P}}\left(\omega / \omega_{1}\right)$, i.e. $k_{1} \simeq$ $.4009375 \cdot 10^{-4}, k_{2} \simeq 4.47686$.

First- and second-order averaged models, and corrected estimation of $\bar{w}(0)$, are defined accordingly. Figure 3 presents a numerical simulation comparing discrete, defined by (24), discontinuous and first-order averaged HSTCP responses.

\section{Conclusions}

We defined discontinuous and averaged models for a class of generalized TCP (GTCP) protocols, which include standard TCP and more recent versions such 
a)
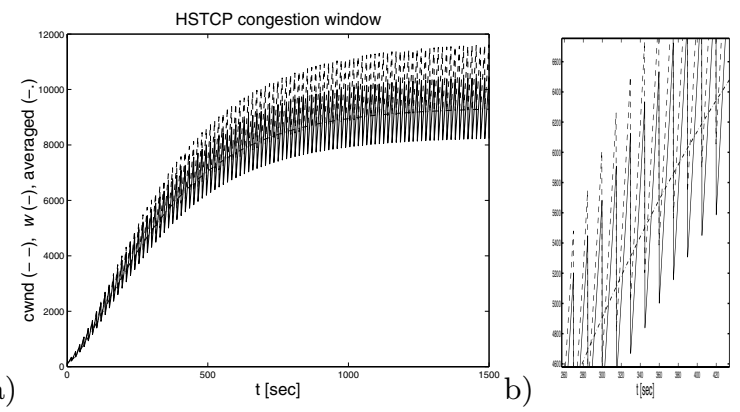

Fig. 3. a) Comparison of discrete (dashed line), discontinuous (solid), and averaged (dash-dotted) HSTCP solutions, initial condition cwnd $=w(0)=\bar{w}(0)=100$ packets, $T=15$ sec, $\mathrm{RTT}=0.2$ sec. b) A zoom.

as HighSpeed TCP. We make the relation between two approaches (discontinuous and averaged) and, under the assumption of fixed RTT and inter-loss time $T$, we show that the second type of models results in fact from the classical, deterministic principle of averaging. We demonstrated uniqueness and stability of periodic behaviors for GTCPs and, particularly, for HighSpeed TCP. Several lines of research will be pursued, including average modeling, conditions of uniqueness, and stability of different types of protocols. It is worth noting, for example, that Scalable TCP [3] yields in our approach a trivial averaged model $(d \bar{w} / d t=0)$. The proposed models can be used to analyze important properties, like bandwidth sharing, and also are useful for control purposes [21].

\section{Acknowledgments}

The authors would like to thank H. Mounier for fruitful discussions on the topics of this paper. This work was supported by an ECOS-Nord grant for FrenchVenezuelan collaboration as well as by an INRIA grant ARC TCP.

\section{References}

1. Chiu, D.M., Jain, R.: Analysis of the increase and decrease algorithms for congestion avoidance in computer networks. Computer Networks and ISDN Systems $\mathbf{1 7}$ (1989) $1-14$

2. Floyd, S., Handley, M., Padhye, J.: A comparison of equation-based and AIMD congestion control. Technical report, ICSI Center for Internet Research (2000) Available at URL: www.icir.org/tfrc/aimd.pdf.

3. Kelly, T.: Scalable TCP: Improving performance in highspeed wide area networks (2002) Submitted for publication.

4. Floyd, S., Ratnasamy, S., Shenker, S.: Modifying TCP's congestion control for high speeds (2002) Preliminary draft. Available at URL:

www.icir.org/floyd/papers/hstcp.pdf. 
5. Baccelli, F., Hong, D.: AIMD, fairness and fractal scaling of TCP traffic. In: Proceedings of IEEE Infocom. (2002)

6. Brown, P.: Resource sharing of TCP connections with diferent round trip times. In: Proceedings of IEEE Infocom, Tel-Aviv, Israel (2000) 151-160

7. Kelly, F.: Mathematical modelling of the Internet. In: Bjorn Engquist and Wilfried Schmid (Eds.), Mathematics Unlimited - 2001 and Beyond. Springer-Verlag, Berlin (2001) 685-702

8. Misra, V., Gong, W., Towsley, D.: Fluid-based analysis of a network of AQM routers supporting TCP flows with an application to RED. In: Proceedings of ACM/SIGCOMM, Stockholm, Sweden (2000) 151-160

9. Altman, E., Avratchenkov, K., Barakat, C., Nunez-Queija, R.: TCP modeling in the presence of nonlinear window growth. In: Proceedings of ITC-17, Salvador da Bahia, Brazil (2001)

10. Misra, V., Gong, W., Towsley, D.: Stochastic differential equation modeling and analysis of TCP windowsize behavior. Technical Report ECE-TR-CCS-99-10-01, Department of Electrical and Computer Engineering, University of Massachusetts (1999) Presented at Performance'99, October Istanbul 1999.

11. Ritter, M.: Network buffer requirements of the rate-based control mechanism for ABR services. In: IEEE Infocom, San Francisco, USA (1996)

12. Low, S.: A duality model of TCP and queue management algorithms. IEEE/ACM Trans. on Networking 11 (2003) 525-536

13. Utkin, V.: Sliding Modes in Control and Optimization. Springer-Verlag, Berlin (1992)

14. Filippov, A.: Differential Equations with Discontinuous Righthand Sides. Kluwer Academic Publishers, Dordrecht (1988)

15. Mahdavi, J., Floyd, S.: TCP-friendly unicast rate-based flow control (1997) Note sent to the end2end-interest mailing list.

16. Altman, E., Barakat, C., Avratchenkov, K.: A stochastic model of TCP/IP with stationary ergodic random losses. In: Proceedings of ACM/SIGCOMM, Stockholm, Sweden, Aug. 28-Sept. 1 (2000)

17. Krein, P., Bentsman, J., Bass, R., Lesieutre, B.: On the use of averaging for the analysis of power electronic system. IEEE Trans. Automat. Contr. 5 (1990) 182190

18. Sanders, J., Verhulst, F.: Averaging methods in nonlinear dynamical systems. Springer-Verlag, New York (1985)

19. Khalil, H.K.: Nonlinear Systems. Prentice-Hall, Upper Saddle River, NJ (2002)

20. Sontag, E.: Mathematical Control Theory: Deterministic Finite Dimensional Systems. Springer-Verlag, New York (1990)

21. Solé-Álvarez, S., Marquez, R., Altman, E.: Control lineal de protocolos para redes de alta velocidad: el caso de HighSpeed TCP. In: Actas (CD-Rom) del IV Congreso de Automatización y Control (CAC'2003), Mérida, Venezuela (2003) 